\title{
VIVER COM DEFICIÊNCIA FÍSICA E O PAPEL DA REDE DE APOIO
}

\section{LIVING WITH PHYSICAL DISABILITY AND THE ROLE OF THE SUPPORT NETWORK}

\section{VIVIR CON DISCAPACIDAD FÍSICA Y EL PAPEL DE LA RED DE APOYO}

Paula Brignol ${ }^{1}$, Soraia Dornelles Schoeller ${ }^{2}$, Denise Maria Guerreiro Vieira da Silva ${ }^{3}$, Julia Estela Willrich Boell ${ }^{4}$, Soraia Geraldo Rozza Lopes $^{5}$, Daniella Karine Souza Lima ${ }^{6}$

\section{RESUMO}

Objetivo: compreender o impacto da deficiência física na pessoa e a contribuição da rede de apoio. Métodos: Trata-se de um estudo exploratório descritivo de abordagem qualitativa. Foram entrevistadas 10 pessoas com deficiência física de um município localizado no Sul do Brasil. Os dados foram coletados no período de março de 2013 a fevereiro de 2015, por meio de entrevistas semiestruturada em profundidade e realizada análise de conteúdo. Resultados: os dados foram organizados em três categorias temáticas: perda da autonomia/independência; mudanças no cotidiano e no viver; e rede como apoio para superar/enfrentar as dificuldades. Conclusão: a deficiência física apresenta transformações na vida das pessoas, sendo a rede de apoio a elas, peça fundamental para o enfrentamento das dificuldades.

Descritores: Enfermagem; Apoio social; Pessoas com deficiência; Reabilitação.

\section{ABSTRACT}

Objective: To understand the impact of physical disability on the person and the contribution of the support network. Methods: This is an exploratory descriptive study with a qualitative approach. Ten people with physical disabilities from a municipality located in the South of Brazil were interviewed. Data were collected from March 2013 to February 2015, through semi-structured in-depth interviews and content analysis. Results: data were organized into three thematic categories: loss of autonomy/independence; changes in daily life and living, and network as support to overcome/face difficulties. Conclusion: the physical disability presents changes in the lives of people, being the network of support to them, fundamental piece to face the difficulties.

Descriptors: Nursing; Social support; Disabled persons; Rehabilitation.

\section{RESUMEN}

Objetivo: Para entender el impacto de la discapacidad física de la persona y la contribución de la red de apoyo. Métodos: Se trata de un estudio cualitativo descriptivo y exploratorio. Hemos entrevistado a 10 personas con discapacidad física en un municipio situado en el sur de Brasil. Los datos fueron recolectados a partir de marzo 2013 a febrero 2015, por meio de entrevistas semiestructuradas y análisis de contenido. Resultados: Los datos se organizan en tres categorías temáticas: la pérdida de la autonomía/independencia; cambios en la vida cotidiana y en el vivir; y la red como apoyo para superar/vencer las dificultades. Conclusión: la discapacidad física presenta cambios en la vida de las personas, la red de apoyo de las personas con discapacidad como una clave para hacer frente a las dificultades.

Descriptores: Enfermería; Apoyo social; Personas con discapacidad; Rehabilitación.

${ }^{1}$ Graduada em Enfermagem. Mestre em Enfermagem pela Universidade Federal de Santa Catarina. ${ }^{2}$ Graduada em Enfermagem. Doutora em Filosofia da Saúde e Enfermagem pela Universidade Federal de Santa Catarina. Docente na Universidade Federal de Santa Catarina. ${ }^{3}$ Graduada em Enfermagem. Doutora em Enfermagem pela Universidade Federal de Santa Catarina. Professora titular, aposentada e docente voluntária do Programa de Pós-Graduação em Enfermagem da Universidade Federal de Santa Catarina. ${ }^{4}$ Graduada em Enfermagem e Educação Física. Doutora em Enfermagem pela Universidade Federal de Santa Catarina. Professora Substituta na Universidade Federal de Santa Catarina. ${ }^{5}$ Graduada em Enfermagem. Doutora em Enfermagem pela Universidade Federal de Santa Catarina. ${ }^{6}$ Graduada em Enfermagem. Pós-Doutorado em Farmacologia de Produtos Naturais pela Universidade Federal de Santa Catarina.

Como citar este artigo:

Brignol P, Schoeller SD, Silva DMGV, et al. Viver com deficiência física e o papel da rede de apoio. Revista de Enfermagem do Centro-Oeste Mineiro. 2018;8:e1957. [Access ; Available in DOI: http://dx.doi.org/10.19175/recom.v7i0.1957 


\section{INTRODUÇÃO}

A deficiência é um termo amplo que compreende as deficiências físicas, as limitações de atividade e a restrição de participação social. Contempla fatores que influenciam a interação entre uma pessoa e seu entorno (ambiente e fatores pessoais). Como tal, é um tema de direitos humanos que obedece ao princípio de que toda pessoa tem o direito de desfrutar de todas as condições necessárias para 0 desenvolvimento de seus talentos e aspirações, sem ser submetido a qualquer tipo de discriminação ${ }^{(1-2)}$. Pessoas com deficiência são aquelas que têm impedimentos de longo prazo de natureza física, mental, intelectual ou sensorial, as quais, em interação com diversas barreiras, podem obstruir sua participação plena e efetiva na sociedade em igualdades de condições com as demais pessoas ${ }^{(1)}$.

Por diversos anos, a deficiência foi entendida apenas como disfunção biológica ou como tragédia por uma irresponsabilidade individual. Somente a partir do fortalecimento do marco dos direitos humanos, da politização de movimentos sociais e da reivindicação dos direitos de cidadania por essa parcela da população é que surgiram diversos esforços para a garantia de direitos adquiridos ${ }^{(3)}$.

De acordo com a Organização Mundial de Saúde, há no mundo uma prevalência de mais de um bilhão de pessoas com algum tipo de deficiência. Desses, cerca de 200 milhões lidam com incapacidades funcionais consideráveis e menos de 50,0\% têm acesso aos cuidados de saúde constitucionalmente adquiridos. No Brasil, há aproximadamente 46 milhões de pessoas com deficiência, sendo 13 milhões de pessoas com deficiência motora ou física, o que corresponde a $6,9 \%$ da população do país. A prevalência de deficiência física é maior no sexo feminino, com cerca de três milhões a mais em mulheres do que em homens ${ }^{(1,4)}$.

As transformações ocorridas na vida das pessoas com deficiência são percebidas em âmbito global, na medida em que elas se vêem de alguma forma menos independentes, podendo provocar ou aumentar as dificuldades que serão proporcionais às limitações que essas apresentarem. A deficiência acarreta mudanças profundas também no funcionamento do organismo e a pessoa necessita de um período de intenso aprendizado, no qual tem que lidar com limitações adquiridas. Essas mudanças demandam adaptações físicas, emocionais e sociais, que determinam o surgimento de novos padrões de vida ${ }^{(5-7)}$.

A pessoa com deficiência física necessita de um ambiente que proporcione condições favoráveis ao seu estado, de forma que ela possa exercer suas atividades, apesar de suas limitações. Este processo de adaptação requer a contribuição e o suporte da rede de apoio, uma vez que a pessoa se sente mais amada e estimada e com sensação de autocontrole. A rede de apoio possibilita a aquisição de informação, desenvolvimento e manutenção do autoconceito, bem como regulação da emoção ${ }^{(8)}$.

Embora haja um grande número de pessoas com deficiência no mundo, são escassos os estudos em saúde voltados ao cuidado de enfermagem para essa população ${ }^{(7)}$, principalmente correlacionando a rede de apoio que as pessoas com deficiência recebem em seu viver. Sendo assim, as pessoas que integram a rede de apoio carecem de informações sobre o seu papel, o apoio prestado e a contribuição dessa nos cuidados e na diminuição dos estressores causados pelas mudanças na saúde e no viver com deficiência. Este estudo percorre justamente essa lacuna de conhecimento, com o objetivo de compreender o impacto da deficiência na pessoa e a contribuição da rede de apoio.

\section{MÉTODOS}

Trata-se de pesquisa qualitativa, realizada num município do Sul do Brasil, no período de março de 2013 a fevereiro de 2015. Os participantes do estudo foram 10 pessoas com deficiência física, selecionados intencionalmente a partir de uma lista fornecida por uma entidade não governamental que presta assistência a essa população. $O$ número de participantes foi definido pelas pesquisadoras a partir da saturação teórica. Os critérios de inclusão foram: pessoa com deficiência física há mais de um ano; maiores de 18 anos; com capacidade de comunicar-se verbalmente. Como critérios de exclusão: Ter alguma deficiência cognitiva, auditiva ou múltiplas deficiências associadas.

A coleta de dados foi realizada por meio de entrevistas em profundidade, semiestruturadas, em dia e horário pré-agendados pelos participantes, que foram contatados por meio telefônico pela lista fornecida. Os questionamentos realizados foram: $\mathrm{O}$ que mudou na sua vida depois da deficiência? Que necessidades a deficiência trouxe para sua vida 
(medicação, dieta/alimentação, atividades físicas/fisioterapia, apoio emocional/afetivo/religioso, consultas com especialistas, adaptações no trabalho, transporte, materiais/equipamentos, adaptações na casa, adaptações no ambiente escolar, lazer, outros)? Quem o apoiou a atender essas necessidades (família, amigos, vizinhos, igreja, centro comunitário, instituição de saúde, grupos específicos, instituições comerciais, centro espírita, terapeutas da comunidade, benzedeira, outros)? As entrevistas foram realizadas no domicílio, com média de duração de 40 minutos e com anuência dos participantes, foram gravadas em formato digital, sendo posteriormente transcritas.

Para a análise dos dados, foram utilizados os pressupostos de Minayo, que se desdobram em três momentos: a pré-análise, que consistiu em leituras aprofundadas das entrevistas; no segundo momento a exploração do material através de pré-codificações dos dados e a apreensão e interpretação dos resultados, propiciando a categorização dos resultados ${ }^{(9)}$.

Além disso, para conferir o anonimato dos participantes, suas identidades foram substituídas pela letra $\mathrm{P}$ seguida por números sequenciais de um a 10 . O estudo respeitou as exigências formais contidas nas normas nacionais e internacionais regulamentadoras de pesquisas envolvendo seres humanos, sob o número 216.396.

\section{RESULTADOS E DISCUSSÃO}

Entre os participantes do estudo, oito deles eram do sexo masculino e dois do sexo feminino, dentre eles, três apresentavam paraplegia causada por acidente automobilístico ou doença crônica; três apresentavam tetraplegia causada por iatrogenia ou mergulho em águas rasas; quatro apresentavam amputação, sendo três em membro inferior e um em membro superior. Desses, quatro eram casados, cinco solteiros e um não informou; entre as ocupações, sete eram aposentados, um era secretário, um era atendente em gráfica e um para-atleta. Em relação aos graus de instrução, um possui ensino superior completo, três possuem superior incompleto, três possuem ensino médio completo, dois com ensino médio incompleto e um ensino fundamental incompleto. O tempo convivendo com a deficiência foi de 7 anos (DP: 4,29).
Quanto aos achados da pesquisa, entre questões importantes relacionadas ao viver com deficiência física, destacamos: perda da autonomia/independência; mudanças no cotidiano e no viver; e rede como apoio para superar/enfrentar as dificuldades que estão descritas a seguir.

\section{Perda da autonomia/independência}

Nesta categoria, os participantes relataram a perda da autonomia ao que se refere às atividades individuais da vida diária que abrangem: vestir-se, cuidados de higiene pessoal, atividades domésticas. São as ações de domínio do próprio corpo, no exercício cotidiano de atividades realizadas anteriormente com facilidade. A execução nas atividades diárias é parte essencial do viver humano e é por meio desta que aprimoramos competências, interagimos com outros e com nossas redes e atribuímos expectativas e significados à vida. As falas a seguir demonstram tais necessidades:

"E tudo para mim era difícil, para lavar uma louça, para lavar uma roupa, para fazer uma comida, hoje se é para ele fazer uma comida ou o que é para cortar ele que faz, ele deixa tudo pronto para mim, eu só". (P 03)

"De início era tudo difícil, eles me pegavam no colo para dar banho, me davam comida praticamente na boca". (P 07)

"Eles faziam tudo para mim, na verdade. Para ir ao médico, eles tinham que me pegar no colo, colocar na cadeira, pegar a cadeira colocar no carro, porque eu não tinha força. Eu ia de maca no início". (P 06)

"Tento ser o máximo independente, mas eu não sou, não adianta. Eu dependo, mas não desisto fácil, muita gente no meu lugar já teria desistido, iria ganhar comida na boca, eu prefiro que não, eu prefiro passar um pouquinho de trabalho, mas tento resolver os meus probleminhas". (P 09)

Percebemos que a autonomia é algo que se torna prejudicada em pessoas com deficiência, justamente pela necessidade de apoio que ela necessita, sendo evidenciado que a rede de apoio oferecida pelos familiares contribuiu na superação e enfrentamento de dificuldades. Nesse contexto, observa-se a perda de autonomia relacionada ao sentimento de dependência e o fato de necessitar de outras pessoas para executar atividades cotidianas. A pessoa com deficiência física sente-se incapaz devido à perda 
da independência e à falta de controle sobre si mesma ${ }^{(10-11)}$.

A perda da autonomia é expressa também pelas mudanças ocorridas. Dentre elas, destacamos: convivência com mudanças corporais, mobilidade reduzida e, muitas vezes, a dependência física e até mesmo psicológica são efeitos avassaladores que as pessoas com deficiência física passam a vivenciar após a constatação de sua atual condição. Percebemos que a deficiência torna as pessoas mais vulneráveis à discriminação social e a barreiras socioambientais, corroborando com os achados destacados neste estudo ${ }^{(12)}$.

A autonomia envolve as atividades cotidianas. Desta forma, a deficiência física estabelece dificuldades que necessitam de planejamento pautado em metas e estratégias a fim de que a pessoa possa enfrentar suas limitações adquiridas, apoiando-se em sua rede, para assim, tornar-se capaz de superar limites e assumir uma posição de controle sobre sua própria vida de maneira autônoma. Esta é uma das tarefas dos profissionais de saúde que atuam no âmbito da reabilitação.

\section{Mudanças no cotidiano e no viver}

Nesta categoria, foi desvelada a necessidade de adaptações no ambiente em que as pessoas com deficiência vivem. As mudanças no viver a partir da deficiência física são os principais relatos dessa categoria. Essas modificações podem ser de diversas fontes, entre elas: utilização de medicamentos, afastamento laboral, internações hospitalares, adaptações necessárias no ambiente intrínseco ao seu lar, bem como nos espaços de circulação coletiva, o que implica em ressignificar sua vida para adaptar-se a sua nova condição física retomando as atividades cotidianas.

"Mudou tudo! Tudo! Tudo! Porque eu trabalhava em dois empregos e ainda estudava a noite". (P 05)

"Sim, sim, remédio que eu não tomava nenhum, eu passei a tomar três, quatro tipos de remédio". (P 03)

"Como ele era marceneiro, percebeu que estava difícil para mim dentro de casa, ele foi e pesquisou com os amigos da empresa, então ele deu um jeito, levantou o piso, arrumou o telhado". (P 07)

"Mudou tudo, tanto é que cai em depressão. Estava fazendo fisioterapia com a Dra. P., como eu tive uma escara, então, eu tive que parar, ficar internado um ano e quatro meses em um hospital, depois mais 15 dias em outro". ( $P$ 08)

As mudanças advindas da nova condição física implicam em adaptações no deslocamento e acesso à ambientes externos, bem como no domicílio da pessoa, sendo necessárias descobertas para retomada da vida social. Como explicitado nos relatos acima, essas pessoas realizam novas ações referentes aos cuidados com sua saúde, por exemplo, o uso de medicamentos contínuos, internações recorrentes e o auxílio de profissionais da saúde para adaptações do ambiente conforme sua nova condição. Desta forma, em decorrência das mudanças geradas pela deficiência, a pessoa sente-se incapaz de viver como antes, pois diversas alterações são experimentadas no cotidiano ${ }^{(6)}$.

Assim, a rede de apoio apresenta-se como peça fundamental para o auxílio na adoção de adaptações e na aquisição de novas habilidades para continuar seguindo a sua vida. Ou seja, sua rede de apoio é fundamental no processo de reintegração e retomada da vida das pessoas com deficiência $^{(13)}$.

\section{Rede como apoio para superar/enfrentar as dificuldades}

Os participantes do estudo tinham, em sua maioria, apoio proveniente de familiares e profissionais de saúde, com diferentes graus de proximidade e distintos tipos de apoio fornecidos.

Nesta categoria, foi evidenciada a presença da rede de apoio e a necessidade desta para o enfrentamento da condição adquirida, além de trazer como atores principais os membros da família e os profissionais e serviços de saúde, evidenciado pelas falas:

"Foi a família...os médicos, principalmente lá do XXX, que eu faço tratamento com eles até hoje (...). O pessoal da enfermagem, a XXX, a XXX. De início foi mais a minha família, nessas horas os amigos somem (...) Então, minha mãe, meus irmãos e irmãs". (P 06)

"Eu tive sorte de ter a minha família que me apoiou muito. Na época em que eu fiquei na cadeira de rodas, minha mãe estava construindo, ela construiu a casa toda adaptada, pensando no depois, ela dizia assim: meu filho não vai ficar na cadeira de rodas, mas se um dia isso acontecer eu quero que ele seja muito bem-vindo na minha casa".(P 07) 
"Meu esposo foi a minha base, ele foi tudo, ele me buscava, ele me levava, ele me dava banho, ele me trocava, ele me dava remédio, ele me dava comida quando eu não conseguia". (P 07).

"O posto de saúde, eles iam lá e faziam o curativo, quando eu estava ruim eu tomava antibiótico e medicação, e eles me ajudavam muito (...), os meus filhos também ajudaram". ( $P$ 08).

Um contraponto se faz necessário destacar, visto que nem todos os participantes desta pesquisa tinham em sua rede de apoio a família e os serviços de saúde. "Agora, eu tenho que me virar com a minha tia. Porque assim, a minha família, a minha mãe os meus irmãos ninguém dá nenhum apoio nenhum deles, não souberam me entender". (P 08)

"Eu tinha a minha sogra, mas a minha sogra quase acabou comigo, quase me matou. Precisou o meu pai falecer, agora dois meses estava no hospital, para a família do meu pai, que não sabia do jeito que eu estava, vir me apoiar". (P 08)

"Algumas coisas eles me davam no serviço de saúde, mas pouca coisa assim, às vezes luvas que precisa para fazer procedimentos assim, eles me davam, mas, não me deram nada, é complicado. Hoje eles dão porque eles foram obrigados a dar". (P 06)

"O serviço público não tem nada. Até medicamento simples, o dipirona, o posto de saúde às vezes não dá, diz que não tem porque é muito barato, aí eu preciso de um medicamento mais caro para bexiga, para controlar a bexiga, aí eles dizem que é caro. Então eles não dão nada na verdade, tudo assim que eu recebo hoje do posto saúde foi porque eu entrei com ação judicial, para poder receber, porque eles não dão nada, é complicado. Não vem me visitar, não recebo nada do órgão público, nada, nada". (P 06)

A rede é vista como forma de superação das dificuldades impostas pela deficiência física. Os relatos trazem a família como suporte principal, porém os profissionais e os ambientes de saúde surgem como parte da rede das pessoas com deficiência, uma vez que a frequência e a duração da terapia reabilitadora tornam possível esse contato. Cabe ressaltar que a ausência de uma rede de apoio eficaz, possivelmente, acaba por prejudicar o enfrentamento da condição.

Neste contexto, Holanda e colaboradores $^{(14)}$, em estudo transversal realizado na atenção primária, buscou identificar a constituição das redes de apoio social das pessoas com deficiência física e as facilidades ao acesso aos serviços de saúde e a inclusão social. 0 estudo demonstrou que as redes estão constituídas, principalmente, pelos componentes da dimensão familiar, onde o apoio contribui para o acesso aos serviços e para a participação em grupos sociais. Sendo a locomoção, a principal barreira para a interação social.

A deficiência física contempla fatores que influenciam a interação entre um indivíduo e seu entorno (fatores pessoais e ambientais) e, como tal, é um tema de direitos humanos que obedece ao princípio de que toda pessoa tem o direito de desfrutar de todas as condições necessárias para o desenvolvimento de seus talentos e aspirações, sem ser submetido a qualquer tipo de discriminação ${ }^{(1-2)}$. Na prática, a garantia dos direitos das pessoas com deficiência exige ações em ambas as frentes, a do direito universal e a do direito de grupos específicos, tendo sempre como objetivo principal minimizar ou eliminar a lacuna existente entre as condições das pessoas com deficiência e as das pessoas sem deficiência.

Em nosso estudo, a família apresentou-se como principal suporte para pessoas nesta condição, no entanto, os profissionais de saúde também foram citados como influenciadores no enfrentamento das pessoas com deficiência física, favorecendo a busca de novas estratégias que viabilizem melhor adaptação a essa condição. Ao analisar o processo de trabalho num Centro de Reabilitação de referência estadual no Sul do Brasil, Schoeller et al. ${ }^{(15)}$, demonstraram que o trabalho multiprofissional facilita o processo de reabilitação das pessoas com deficiência, trazendo benefícios de forma singular aos usuários alvo desta abordagem, e que o trabalho de enfermagem é parte essencial desse processo. A atuação dos profissionais de saúde é percebida como de extrema importância para a pessoa com deficiência, já que é capaz de proporcionar um novo caminho de recuperação da autonomia individual, ao mesmo tempo em que auxilia nas mudanças ambientais necessárias ${ }^{(16-17)}$.

A deficiência física coloca a pessoa frente a uma multiplicidade de desafios físicos, psicológicos e sociais, podendo trazer prejuízos nesses aspectos, bem como alterações na imagem corporal e no autoconceito. Porém, é preciso considerar que há variação na percepção psicossocial de pessoas com deficiência física. Muitos conseguem enfrentar, de forma positiva, a condição vivenciada, principalmente quando se sentem amparados por uma rede de apoio; 
porém, para outros, a rede acaba por não oferecer suporte adequado, o que dificulta a criação de estratégias de enfrentamento ${ }^{(15-17)}$.

Esta pesquisa aponta para questões importantes relacionadas à rede de apoio às pessoas com deficiência, elencando os principais elementos da mesma e sua contribuição à saúde destas pessoas. No entanto, o número de participantes reduzido constitui uma limitação do mesmo, fato que coloca a necessidade de outros estudos com a mesma temática. Acresça-se a isso o pequeno número de investigações sobre a rede de apoio a esta parcela populacional.

O presente estudo aponta aspectos relevantes relacionados ao viver com a deficiência física. A enfermagem torna-se responsável por uma contribuição prática e teórica, que proporcione diferentes visões, que auxilie as pessoas com deficiência em seu enfrentamento. Assim, os profissionais de saúde devem assumir seu papel como fortalecedor dos vínculos apoiadores e da rede de apoio, tornando-se participantes ativos desta, buscando conhecer a natureza das relações e como isso tende a auxiliar na reabilitação e reinserção dessas pessoas na sociedade.

O número de participantes reduzido constituiu uma limitação do estudo, fato que coloca a necessidade de outros estudos com a mesma temática. Acresça-se a isso o pequeno número de investigações sobre o viver com deficiência, bem como a rede de apoio dessa parcela populacional. Novas investigações são indispensáveis no que tange à deficiência física e ao apoio recebido para a busca pela autonomia e o enfrentamento da condição. E ainda apontamos para a necessidade de estudos que relacionem as condições de vida com o enfrentamento e as possibilidades de adaptação para o viver com deficiência.

\section{CONCLUSÃO}

Compreender como vivem as pessoas com deficiência física ultrapassa os limites biológicos, pois precisamos considerar aspectos psicológicos, sociais e culturais, ou seja, transcender a questão clínica, pois a pessoa é o reflexo de diversos fatores que a influenciam e que fazem parte de seu cotidiano.

A deficiência física apresenta transformações imensuráveis na vida dos que a adquirem, e é nesse caminho que os achados deste estudo transitam. Dentre as necessidades mais visíveis, antes não conhecidas pelas pessoas e o convívio com sua condição, está a adaptação do ambiente externo e interno, geralmente inapropriados para recebê-los, o que dificulta sua reinserção na sociedade e o enfrentamento de sua nova condição.

A ação da rede de apoio, tanto familiar quanto profissional, junto a pessoas com deficiência é de suma importância, visto que essa apresenta diversas demandas, tanto de cuidados terapêuticos, como de apoio psicológico, promovendo melhoria na qualidade da vida, além de auxiliar significativamente em todo o processo de reabilitação.

\section{REFERÊNCIAS}

1. Organização Mundial de Saúde (OMS). Relatório mundial sobre a deficiência [Internet]. São Paulo: SEDPCD; 2012. Disponível em: http://www.pessoacomdeficiencia.sp.gov.br/usr/ share/documents/RELATORIO MUNDIAL COMPL ETO.pdf

2. Ministério da Saúde (Brasil), Secretaria de Direitos Humanos da Presidência da República, Secretaria Nacional de Promoção dos Direitos da Pessoa com Deficiência, Coordenação-Geral do Sistema de Informações sobre a Pessoa com Deficiência. Cartilha do censo 2010: pessoas com deficiência [Internet]. Brasília, DF: SDH-PR/SNPD; $2012 . \quad$ Disponível em: http://www.pessoacomdeficiencia.gov.br/app/sit es/default/files/publicacoes/cartilha-censo-2010pessoas-com-deficienciareduzido.pdf

3. Santos WR. Deficiência e BPC: o que muda na vida das pessoas atendidas? Ciênc Saúde Coletiva [Internet]. 2011 [citado em 2017 ago 8];16(Suppl 1):787-96. Disponível em: http://www.scielo.br/scielo.php?script=sci arttex $\mathrm{t} \& \mathrm{pid}=\mathrm{S} 1413-81232011000700009 \& \ln \mathrm{g}=\mathrm{en}$

4. Ministério da Saúde (Brasil), Ministério do Planejamento, Instituto Brasileiro de Geografia e Estatística. Censo demográfico 2010: características gerais da população, religião e pessoas com deficiência. Brasília, DF; 2010. Disponível em: http://www.ibge.gov.br/home/estatistica/populac ao/censo2010/caracteristicas religiao deficiencia/ default caracteristicas religiao deficiencia.shtm

5. Schoeller SD, Bitencourt RN, Leopardi MT, Pires DP, Zanini MTB. Mudanças na vida das pessoas com lesão medular adquirida. Rev Eletr Enferm [Internet]. 2012 [citado em 2016 nov 24];14(1):95-103. Disponível em: http://www.fen.ufg.br/revista/v14/n1/pdf/v14n1 a11.pdf 
6. Trierveiler KS, Ramos FRS, Schoeller SD, Nogueira GC, Pereira FS, Martins MM, et al. Funcionalidade familiar da pessoa com lesão medular. Texto Contexto Enferm [Internet]. 2015 [citado em 2017 ago 8];24(4):993-1002. Disponível em: http://www.scielo.br/scielo.php?script=sci arttex t\&pid=S0104-07072015000400993\&lng=en

7. Yamashita CH, Gaspar JC, Amendola F, Alvarenga MRM, Oliveira MAC. Rede social de cuidadores familiares de pacientes com incapacidades e dependência. Rev Esc Enferm USP [Internet]. 2014 [citado em 2018 mar 2];48(1):97-103. Disponível em: http://www.scielo.br/pdf/reeusp/v48nspe/pt 00 80-6234-reeusp-48-esp-097.pdf

8. Minayo MCS. Análise qualitativa: teoria, passos e fidedignidade. Ciênc Saúde Coletiva [Internet]. 2012 [citado em 2018 mar 2];17(3):621-6. Disponível em: https://social.stoa.usp.br/articles/0028/5691/Mi nayo MCS 2012.pdf

9. Borges AMF, Brignol P, Schoeller SD, Bonetti A. Percepção da pessoa com lesão medular sobre sua condição. Rev Gaúcha Enferm [Internet]. 2012 [citado em 2017 ago 8];33(3):119-25. Disponível em: http://www.scielo.br/scielo.php?script=sci arttex t\&pid=S1983-14472012000300016\&lng=en

10. Brunozi $A E$, Silva $A C$, Gonçalves $L F$, Veronezi RJB. Qualidade de vida na lesão medular traumática. Rev Neurocienc [Internet]. 2011 [citado em 2017 nov 2];19(1):139-44. Disponível em:

http://www.revistaneurociencias.com.br/edicoes 2011/RN1901/revisao/444\%20revisao.pdf

11. Schoeller SD, Grumann ARS, Martini AC, Forner S, Sader LT, Nogueira GC. Conhecer para cuidar características de pessoas com lesão medular atendidas em um centro de reabilitação. Fisioter Mov [Internet]. 2015 [citado em 2017 ago 8];28(1):77-83. Disponível em: http://www.scielo.br/scielo.php?script=sci arttex $\underline{\text { t\&pid}=S 0103-51502015000100077 \& \operatorname{lng}=e n}$

12. Gaudenzi P, Ortega F. Problematizando o conceito de deficiência a partir das noções de autonomia e normalidade. Ciênc Saúde Coletiva [Internet]. 2016 [citado em 2017 abr 20];21(10):3061-70. Disponível em: http://www.scielo.br/scielo.php?script=sci arttex $\underline{\text { t\&pid}=S 141381232016001003061 \& \operatorname{lngt}}$

13. Forouzan AS, Mahmoodi A, Shushtari ZS, Salimi Y, Sajjadi H, Mahmoodi Z. Perceived social support among people with physical disability.
Iran Red Crescent Med J [Internet]. 2013 [citado em 2016 ago 27];15(8):663-7. Disponível em: https://www.ncbi.nlm.nih.gov/pmc/articles/PMC 3918189/pdf/ircmj-15-663.pdf

14. Holanda CMA, Andrade FLJP, Bezerra MA, Nascimento JPS, Neves RF, Alves SB, et al. Support networks and people with physical disabilities: social inclusion and access to health services. Ciênc Saúde Coletiva [Internet]. 2015 [citado em 2017 ago 10];20(1):175-84. Disponível em:

http://www.scielo.br/scielo.php?script=sci arttex t\&pid=S1413-81232015000100175

15. Schoeller SD, Bento LM, Lorenzetti J, Klein AC, Pires D. Processo de trabalho em reabilitação: a perspectiva do trabalhador e do usuário. Aquichán [Internet]. 2015 [citado em 2017 ago 8];15(3):403-12. Disponível em: http://www.scielo.org.co/scielo.php?script=sci ar ttext\&pid=S1657-59972015000300008\&lng=en

16. Andrade LT, Araújo EG, Andrade KRP, Soares DM, Ciancai TCM. Papel da enfermagem na reabilitação física. Rev Bras Enferm [Internet]. 2010 [citado em 2017 ago 8];63(6):1056-60. Disponível

em:

http://www.scielo.br/scielo.php?script=sci arttex t\&pid=S0034-71672010000600029\&lng=pt

17. Rodrigues JSM, Ferreira NMLA. Estrutura e funcionalidade da rede de apoio social do adulto com câncer. Acta Paul Enferm [Internet]. 2012 [citado em 2017 ago 8];25(5):781-7. Disponível em: http://www.scielo.br/scielo.php?script=sci arttex t\&pid=S0103-21002012000500021\&lng=en

Nota: Este estudo é produto do macroprojeto intitulado "A condição do deficiente físico em Florianópolis: perfil epidemiológico, qualidade de vida, redes de apoio e processo de trabalho", financiado pelo Programa de Apoio a Núcleos de Excelência, vinculado à Fundação de Amparo à Pesquisa e Inovação do Estado de Santa Catarina e faz parte da dissertação de Mestrado intitulada "Rede de apoio à pessoa com deficiência física", apresentada ao Programa de Pós-graduação em Enfermagem da Universidade Federal de Santa Catarina.

Recebido em: 03/05/2017

Aprovado em: 12/04/2018

Endereço de correspondência:

Soraia Geraldo Rozza Lopes

Rua José João Martendal, 185, Carvoeira, CEP: 88040-420 - Florianópolis/SC - Brasil

E- mail: soraia.gr.lopes@gmail.com 